\title{
Comparison and Evaluation of the T-Lohi MAC for Underwater Acoustic Sensor Networks
}

\author{
Affan A. Syed, Student Member, IEEE, Wei Ye, Member, IEEE, John Heidemann, Senior Member, IEEE
}

\begin{abstract}
This paper introduces T-Lohi, a new class of distributed and energy-efficient media-access protocols (MAC) for underwater acoustic sensor networks (UWSN). MAC design for UWSN faces significant challenges. For example, acoustic communication suffers from latencies five orders-of-magnitude larger than radio communication, so a naive CSMA MAC would require very long listen time resulting in low throughput and poor energy efficiency. In this paper, we first identify unique characteristics in underwater networking that may affect all MACs, such as space-time uncertainty and deafness conditions. We then develop T-Lohi employing a novel tone-based contention resolution mechanism that exploits space-time uncertainty and high latency to detect collisions and count contenders, achieving good throughput across all offered loads. T-Lohi exploits a low-power wake-up receiver to significantly reduce energy consumption. We evaluate design choices and protocol performance through extensive simulation. Finally, we compare T-Lohi against a few canonical MAC protocols. The results show that the energy cost of packet transmission is within 3-9\% of optimal, and that Lohi achieves good channel utilization, within $30 \%$ utilization of the theoretical maximum. We also show that Lohi is stable and fair under both low and very high offered loads. Finally, we compare Lohi with other alternatives, including TDMA, CSMA, and ALOHA. Except for TDMA under heavy load, Lohi provides the best utilization in all cases, and it is always the most energy efficient.
\end{abstract}

Index Terms-Computer networks, Underwater acoustic communication, Underwater acoustics, Wireless sensor networks, Underwater communication, Underwater technology, Access protocols, Acoustic applications, Protocols.

\section{INTRODUCTION}

$\mathbf{N}$ ETWORKS with shared media require access protocols (MACs) to control access of the shared channel. In underwater sensornets (UWSN), a shared acoustic medium raises challenges absent from traditional RF wireless [1], [2]. Acoustic communication magnifies wireless bandwidth limitations, transmit energy costs, and variations in channel propagation. Control algorithms of MAC protocols are significantly changed by acoustic propagation latencies that are five orders of magnitude greater than radio.

The focus of this paper is to design an energy and throughput efficient MAC protocol for dense and short range acoustic sensor networks. While a vast majority of existing

Manuscript received February 5, 2008; revised July 15, 2008. This research is partially supported by the National Science Foundation (NSF) under the grant NeTS-NOSS-0435517 as the SNUSE project and grant number CNS0708946, "Open Research Testbed for Underwater Ad Hoc and Sensor Networks", and by Chevron Co. through USC Center for Interactive Smart Oilfield Technologies (CiSoft).

Affan Syed, Wei Ye and John Heidemann are with the Information Sciences Institute at the University of Southern California, Marina Del Rey, CA, 90292, USA (e-mail:\{asyed,weiye,johnh\}@isi.edu).

Digital Object Identifier 10.1109/JSAC.2008.081212. underwater networking applications demand sparse and long range deployments, our earlier work motivates a host of promising sensornet-like applications [1]. Recently several innovative acoustic modems have been proposed [3], [4], but MAC protocols have not yet been proposed that exploit their unique low-power capabilities. We will show that the challenges of high latency also enable new MAC techniques and solutions that provide good throughput across varying application requirements (Section II-A).

Many underwater applications will require long-term deployment, making energy-efficient design an important goal. These include static applications such as 4-D seismic sensing of oilfields [1], gliders and low-energy mobile platforms, or platforms with parasitic mobility, such as tagging of aquaticlife [5]. Compared to radio communications, underwater acoustic networking presents different design trade-offs, since transmit energy costs are higher [2], idle times are longer, and battery replacement is harder.

We propose a new class of MAC protocols called Tone Lohi ("Lohi" means slow in Hawaiian). Besides being energy and throughput efficient, Tone Lohi (T-Lohi) provides flexible, fair and stable medium access for acoustic networks. T-Lohi is designed for general underwater applications (not a specific application [6]), and conserves energy through a tone-based contention algorithm with low-power wake-up hardware.

This paper provides three novel contributions. First, we exploit the space-time uncertainty effect [7] to provide contender counting, and show how contender counting can improve fairness and provide throughput stability under high load (Section II-A). Second, we present T-Lohi, a new class of MAC protocols for underwater acoustic networks that utilizes contender counting and low-power wake-up capability of acoustic modems (Section III). Finally, we validate the design decisions behind T-Lohi flavors (Section V-A) and compare T-Lohi to several canonical medium access mechanisms (Section V-C).

To understand T-Lohi performance we perform extensive simulations (Section IV). Our channel model is quite simple; ignoring any channel related packet loss and multipath to focus on protocol performance. Our results are a good preliminary indication of the viability of our MAC and considering channel effects is a very promising future research area. We discuss these assumption and their impact on our MAC in Section IV-F. Our simulations show that T-Lohi is energy efficient within 3-9\% of optimal, and can achieve utilization within $30 \%$ of the theoretical optimal channel capacity. We also show that our protocols are stable and fair under both low and very high offered loads (Section IV-B1). We deepen our previous evaluations [8] by considering different lengths of 


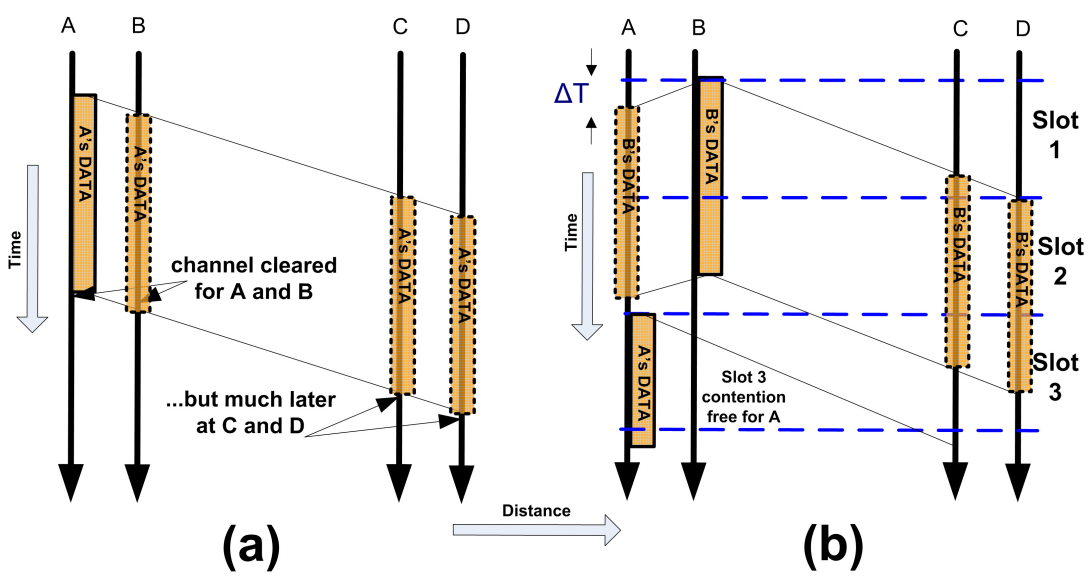

Fig. 1. Spatial Unfairness: (a) Transmitter and close neighbors have channel cleared earlier. (b) In slotted access, close neighbor A can attempt in slot 3 while $\mathrm{C}$ and $\mathrm{D}$ can not.

the contention duration in T-Lohi and confirming our selected values (Section V-A). Moreover, we evaluate design choices with T-Lohi flavors (Section V-B), and compare T-Lohi with representative MAC protocols, such as TDMA, CSMA and ALOHA. Such comparison improves our understanding of when and where T-Lohi is the best choice for medium access (Section V-C). Overall, our results are promising and suggest further evaluation in multi-hop conditions and field tests as promising future directions.

\section{Challenges And Opportunities}

Prior work outlines challenges inherent to underwater acoustic communications [1], [2]; of these challenges, the propagation latency $(200,000$ times longer than radio) has the greatest impact on networking protocols. Acoustic modems also have very different energy consumption patterns compared to radios, with transmission often 100 times more expensive than reception [2]. For example the typical receive:transmit power ratio of WHOI micro-modem is 1:125 [3], while short-range radios for sensornets generally provide ratios around 1:1.5 [9]. Several acoustic modems have a low-power idle mode that draws considerably lower power than either receive or transmit mode [4], [10], [3]. As we will show, our design exploits this capability for energy-efficient medium access.

The simulation results in this paper and our MAC design assumes the specifications of our SNUSE modem [4], transmitting at 1 kbaud in the $17-19 \mathrm{kHz}$ frequency range with FSK encoding. This modem has an expected range of 50$500 \mathrm{~m}$ with a sub-mW wake-up receiver (power specifications provided in Table I). We have done in-water tests of data transmission with this modem, and our implementation of TLohi over this hardware is in progress. Although designed for this modem, we expect T-Lohi to operate with any modem providing wake-up capabilities.

These unique characteristics create behavior in acoustic networks that are not seen in radios. We next describe these characteristics and how we can exploit them to improve MAC design.

\section{A. Space-Time Uncertainty}

Channel state in short-range RF networks can be estimated quickly since propagation delay is negligible. The large propagation delay of acoustic media makes it essential to also consider the locations of a receiver and potential interferers. Distance between nodes translates into uncertainty of current global channel status: space-time uncertainty. Although prior underwater work implies this uncertainty [11], we were the first to explicitly describe it [7], and here we quantify its impact on medium access specific issues and exploit it in our MAC design.

A significant impact of space-time uncertainty is an inherent bias for medium access that depends on location. We call this bias spatial unfairness; it is conceptually similar to channel capture [12], but caused by physical location and propagation latency rather than backoff estimates. Since a packet's arrival time is proportional to distance from transmitter, the channel becomes clear earlier at nodes closer to the transmitter. In Figure 1(a) transmitter $\mathrm{A}$ and its close neighbor $\mathrm{B}$ have a greater chance to recapture the channel after sending than nodes $\mathrm{C}$ and $\mathrm{D}$ that are far away. With slotted media access spatial unfairness becomes more pronounced. In Figure 1(b), B's data ends in slot 2 for nodes A and B, but ends in slot 3 for $C$ and $D$. Thus, even if the transmitter is prevented from immediately reacquiring the channel, nodes A and B can swap the channel back and forth. We handle spatial unfairness in our protocol design by employing a distributed backoff mechanism (Section III-B).

Although latency increases uncertainty, we next show that it can also be exploited for contender detection (CTD) and contender counting (CTC). Nodes in our protocol detect contenders by listening to the channel after sending short reservation tones that are analogous to RTS messages. Unlike lowlatency wireless protocols, large propagation delays allows observation of tones sent concurrently because they may arrive after their own transmissions complete. Contender detection depends on relatively short tones and a long listen period. Nodes can further count the number of contenders, if tones are short enough (we formalize shortness in Section II-B), since tones from different transmitters arrive at different times due to varying propagation latencies. An example is shown in 


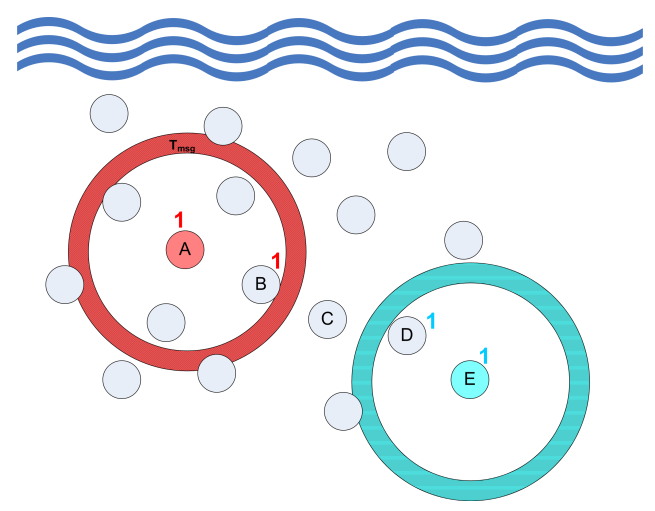

(a) Collision uncertainty

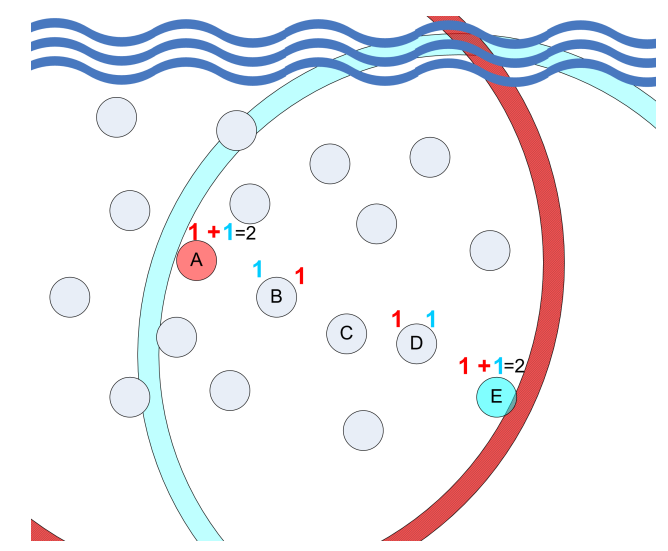

(b) Contender detection and counting

Fig. 2. Spatio-temporal uncertainty in acoustic medium access.

Figure 2(b), where nodes A and E send short tones. At nodes where the tones do not collide, such as nodes A, E, B, and D, they can count the number of contenders. Even if the tones collide on some nodes, e.g., node $\mathrm{C}$, they can still detect the presence of contention. The capability of contender counting (CTC) is not generally possible for RF-based networks due to short propagation delays, although concurrent with our work, some researchers have begun to use game theoretic approximations of contender counts [13]. We exploit CTC in our MAC design in Section III. Others have proposed flowlevel contention counting for multi-hop 802.11 networks [14]; our work differs by focusing on single-hop contention as applied to MAC protocols.

\section{B. Deafness Conditions}

Wireless transceivers normally work in half-duplex mode, and thus on a single channel a node that is transmitting cannot receive another packet at the same time. Therefore, a node becomes deaf to another transmission when transmitting. Deafness is important because nodes need to coordinate access to the medium. We use a custom wake-up circuit and tones for coordination [4]. To achieve very low energy consumption, this circuit must accumulate energy to activate. Because the modem is half-duplex, energy does not begin to accumulate until it starts listening. The minimum time to accumulate activation energy, $T_{\text {detect }}$, represents an additional period of deafness around any transmission time, since tones sent in that period will otherwise not be successfully detected.

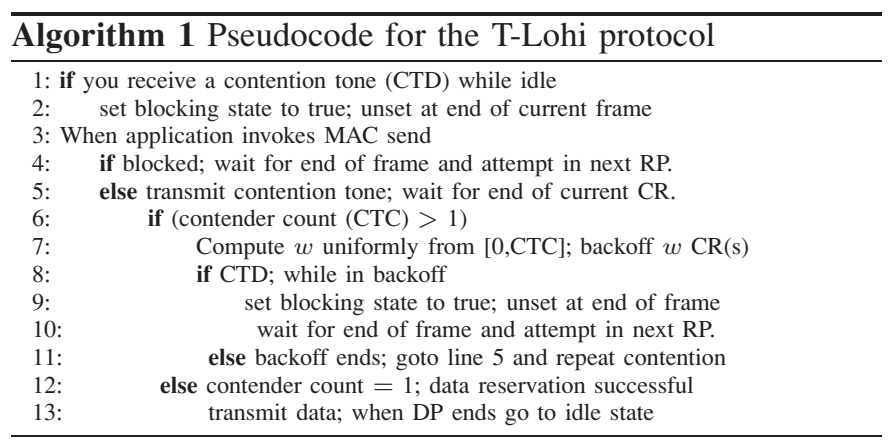

We next explore how latency of tone detection relates to deafness conditions. Three different circumstances can result in deafness. In Figure 3(a), the deafness is bidirectional, as neither node A nor B can detect the other's tone. Figure 3(b) and (c) show two scenarios that lead to unidirectional deafness, where only node B cannot detect A's tone.

We posit that the deafness conditions can be generalized if we make the convention that node A transmits its tone before B. We define TDT (Time Difference of Transmission) as $t_{t x, B}-t_{t x, A}\left(t_{t x, B}\right.$ is the global tone transmit time of $\left.\mathrm{B}\right)$, and $T D L$ (Time difference of Location) as $T_{A, B}\left(T_{A, B}\right.$ is the propagation delay between A and B). Then the Generalized Deafness Condition (GDC):

$$
|T D T-T D L|<T_{\text {detect }}
$$

GDC emphasizes that deafness is not affected by the tone length, but only by tone detection time (for equal length tones). This independence is because tones provide only positive or negative information. The GDC also reflects the space time uncertainty by the dependence of deafness on both relative location (TDL) and transmit time (TDT). In Section III-C, we explore the impact of deafness condition on our MAC protocol.

\section{Tone-Lohi MAC PRotocol Design}

In this section we describe T-Lohi in detail, including its motivation and the design tradeoffs behind different flavors of T-Lohi. We end by identifying the underlying assumptions and limitations of the current T-Lohi design.

\section{A. Overview of T-Lohi}

The primary objective of T-Lohi is to provide a MAC protocol that has efficient channel utilization, stable throughput, and low energy consumption. By its use of contention to reserve the channel, it provides efficient channel utilization and throughput stability. This reservation prevents data packet collision (or makes them very unlikely), thus avoiding loss of throughput and energy waste. It also exploits our modem's very-low-power wake-up tone receiver [4].

In T-Lohi, nodes contend to reserve the channel to send data. (Pseudocode for the T-Lohi protocol is shown as Algorithm 1.) Figure 4 shows this process: each frame consists of a reservation period (RP) followed by a data transfer. Each $\mathrm{RP}$ consists of a series of contention rounds (CRs) until one node successfully reserves the channel. While not shown in the figure, the frame length is provided in the data header, allowing nodes to compute the end-of-frame. 


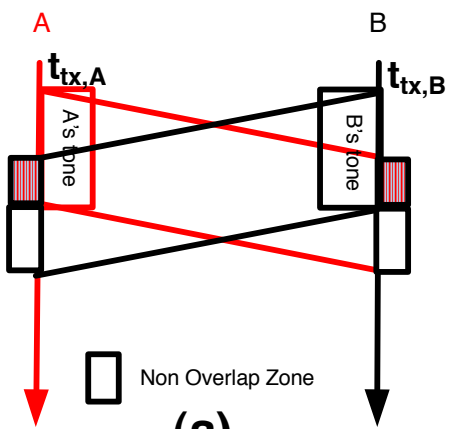

(a)

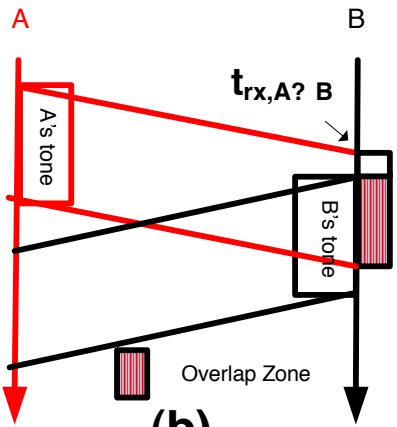

(b)

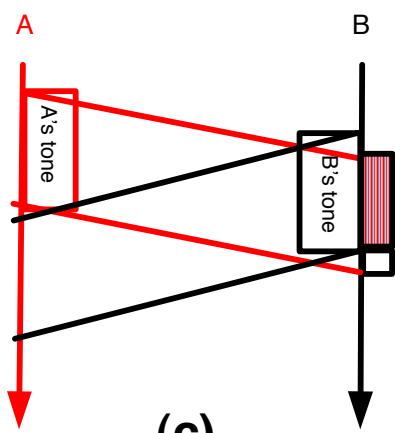

(c)

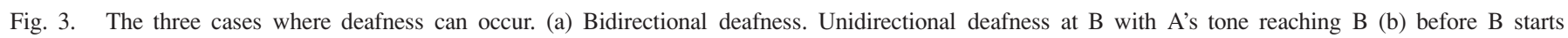
transmitting, (c) after B starts transmitting.

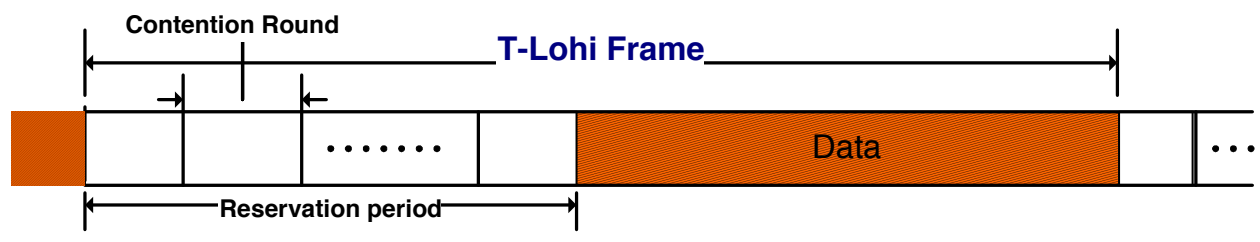

Fig. 4. The Tone-Lohi protocol frame

Contention requires that nodes first send a short tone and then listen for the duration of the contention round (CR) to decide if reservation is successful. If only one node contends in a CR, it wins, ending the RP and then transmitting its data. When nodes detect contention (Algorithm 1, line 7), they randomly back-off in proportion to the contender count, extending the RP. Random backoff promotes fairness, while the window size equal to contender count allows quick convergence based on current load. The $\mathrm{CR}$ is long enough to allow nodes to detect and count contenders (CTD and CTC).

T-Lohi uses our custom, low-power, wake-up tone receiver to conserve energy [4]. Wake-up tones share the channel with data transmissions, but detecting a tone consumes only $2 \%$ the energy of listening for data. Transmitters send a wake-up tone before any data transmission, allowing receivers to keep their $\mathrm{CPU}$ and data receiver off. Powering off transmit and receive and using our low-power wake-up circuit are essential to reduce energy consumption, since correct estimate of channel state requires channel awareness for times on the order of propagation delays (large fractions of a second). A larger potential source of savings follows because T-Lohi's reservation mechanism can prevent data collisions and avoid expensive (re)transmissions. We also suppress successive transmissions from a successful sender to reduce spatial unfairness (Section II-A). The exact duration of this quiet time depends on the T-Lohi variants to be discussed in Section III-B.

\section{B. T-Lohi Flavors}

The T-Lohi reservation mechanism deals with how nodes contend for the channel and make their decisions on channel acquisition by taking the space-time uncertainty into consideration. The backoff mechanism dictates the reaction to a failed contention round as well as the policy to start contention in a new T-Lohi frame, leveraging information about medium access such as CTC. We next define three flavors of T-Lohi that vary the reservation mechanism with different implementation requirements and performance results. (In Section IV-E we also vary the backoff mechanism.)

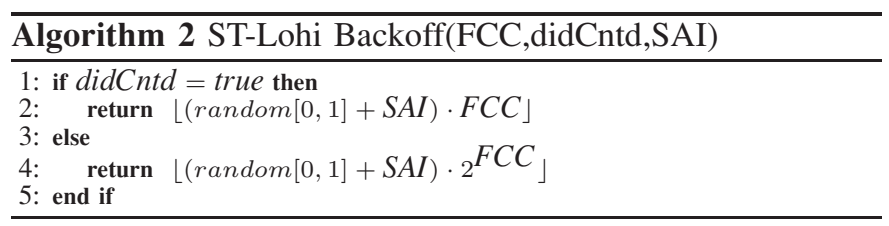

1) Synchronized T-Lohi (ST-Lohi): We begin by assuming all nodes are time synchronized and present ST-Lohi. Synchronizing each contention round simplifies reasoning about protocol correctness, at the cost of requiring distribution of some reference time.

ST-Lohi synchronizes all communication (contention and data) into slots. This duration of contention round is $C R_{S T}=$ $\tau_{\max }+T_{\text {tone }}$, where $\tau_{\max }$ is the worst case one-way propagation time and $T_{\text {tone }}$ is the tone detection time. Figure 5(a) shows ST-Lohi in action, where two nodes contend in the first $\mathrm{CR}$, one in the second $\mathrm{CR}$, then the winner starts sending data in the third slot.

Since tones are sent only at the beginning of each $\mathrm{CR}$, we know that any tones must arrive before the end of the $\mathrm{CR}$ and will be detected assuming no bidirectional deafness (Section II-B). Since bidirectional deafness happens deterministically based on node location (and only rarely when nodes are extremely close), ST-Lohi contention will always converge and provide collision-free data transfer.

Synchronization also provides information about the approximate number of nodes with data to send. We call this value the first contender count (FCC). FCC is updated if in any $\mathrm{CR}$ the CTC is greater than the current $F C C$ and decremented after each frame. In addition, all nodes can estimate the distance from a transmitter by measuring the 


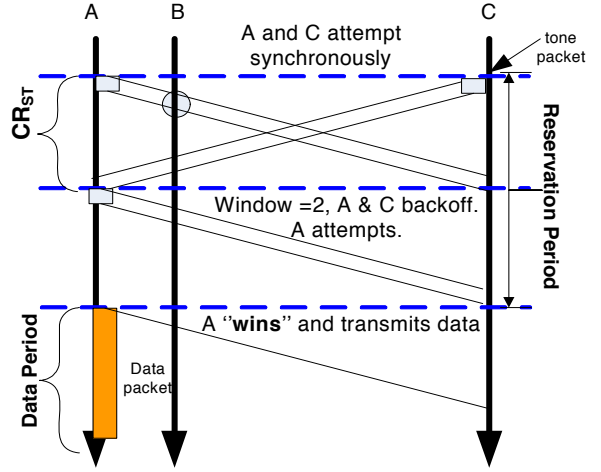

(a)

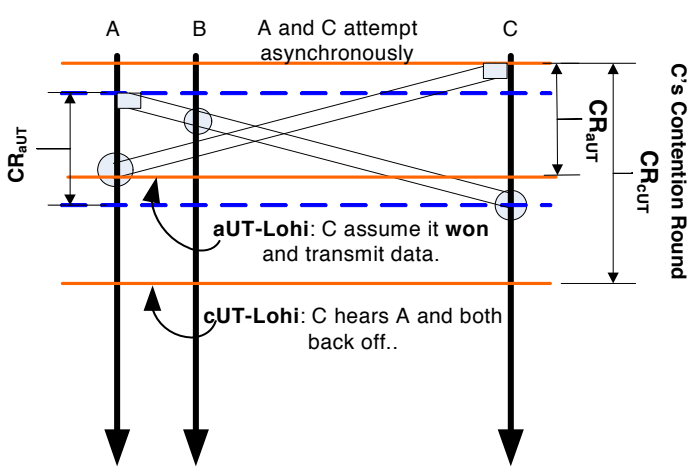

(b)

Fig. 5. Overview of (a) ST-Lohi, (b) UT-Lohi

propagation delay relative to the start of the current slot $(\Delta T$ in Figure 1(a)). We use $\Delta T$ to compute a spatial advantage index, $S A I=1-\frac{\Delta T}{C R_{S T}}$. Nodes also maintain a boolean variable didCntd indicating if they attempted contention in a previous frame. This variable is reset every time node wins the frame and sends data.

Algorithm 2 shows ST-Lohi's backoff algorithm using the SAI and the didCntd flag. After contending, nodes prioritize their channel access, thus reducing the medium access latency. Nodes with higher SAI are more likely to wait an extra slot, which also reduces potential unfairness that can result in channel monopoly between spatially nearby nodes (see Section II-A and Figure 1(b)).

2) Conservative Unsynchronized T-Lohi (cUT-Lohi): STLohi is simple to reason about and we can exploit synchronization to estimate contender behavior. However, time synchronization is not free, and maintaining time synchronization adds run-time overhead and protocol complexity. We therefore next explore unsynchronized protocols.

In unsynchronized T-Lohi, nodes can start contending any time they know the channel is not busy. To provide the same contention detection guarantee as ST-Lohi, cUT-Lohi must observe the channel for $C R_{c U T}=2 \tau_{\max }+2 T_{\text {tone }}$. Consider Figure 5(b), where node $\mathrm{C}$ sends a tone at time $t_{C}$. In the worst case, the second contender $\mathrm{A}$ sends its tone at $t_{C}+\tau_{\max }+T_{\text {tone }}-\epsilon$ because it is as far from $\mathrm{C}$ as possible and sends just before hearing C's tone, and A's tone will arrive and be detected at $\mathrm{C}$ at $t_{C}+2 \tau_{\max }+T_{\text {tone }}-\epsilon$. Unlike ST-Lohi, cUT-Lohi cannot estimate a variable similar to FCC because of an asynchronous view of a contention round, it therefore defaults to just the quite period of a single CR duration after each transmission.

3) Aggressive UT-Lohi (aUT-Lohi): Although cUT-Lohi avoids the complexity of synchronization, its long contention time reduces throughput. Aggressive unsynchronized T-Lohi (aUT-Lohi) follows cUT-Lohi, but cuts the duration of its contention round to $C R_{a U T}=\tau_{\max }+T_{\text {tone }}$.

The purpose of the long listen in cUT was to account for worst-case timing of tones. In aUT-Lohi, worst-case timing results in either a tone detection (as before), or a tone-data collision or data-data collision, depending on the relative distances of the two senders and a receiver. Consider Fig- ure 6(b): B's tone will not be heard by A within $C R_{a U T}$, so A will assume it has acquired the channel and transmit data at $t_{t x, C}+C R_{a U T}$. B's tone and A's data transmissions will collide at a node located within the shadow region near A (a tone-data collision), but be received separately at other nodes. Also, node B will hear A's tone and backoff. We describe these scenarios in more detail in Section III-C1, arguing that the conditions that result in data collisions are quite unlikely. Simulation results in Section IV-D verify the low probability of such events as there are few packet losses for aUT-Lohi.

\section{Discussion on Protocol Correctness}

T-Lohi avoids packet collisions through a reservation mechanism. However, deafness and aggressive contention can cause the reservation mechanism to fail and lose packets. We next define conditions that lead to incorrect reservation, protocol incorrectness, and can cause packet loss. These cases include tone-data collision, data-data collision and persistently incorrect reservation. We also discuss how higher contention can lead to partially correcting these problems.

1) Tone-Data Collision: As described above in Section III-B3, tone-data collision can occur in aUT-Lohi because contenders listen for only $\tau_{\max }$. (It also occurs in very unlikely corner cases with cUT-Lohi and ST-Lohi.) The necessary conditions for tone-data collision in aUT-Lohi are:

\section{Tone-Data coexistence conditions:}

$$
T D T<\left(T D L+T_{\text {tone }}\right) ; \quad T D L \geq \tau_{\max } / 2
$$

The left inequality states that the interferer B must transmit before A's tone is detected by $\mathrm{B}$, as tone detection precludes any contention attempt. (see Figure 6(b)). This condition is a superset of the deafness condition, so if deafness occurs, it will be satisfied, but not vice versa. The second equation represents the case that B is located far enough from A so that the $\mathrm{CR}$ at $\mathrm{A}$ ends before $\mathrm{A}$ can detect the tone sent by $\mathrm{B}$.

However these conditions are not sufficient for tone-data collision. The overlap of tone-data must occur at the receiver (within the shadow region of A as shown in Figure 6(b)) for an actual tone-data collision. This additional condition makes tone-data collision less likely to occur; (also supported by the very small number of tone-data packet losses in simulations in Section IV-D). In fact, if the receiver is not in the shadow 


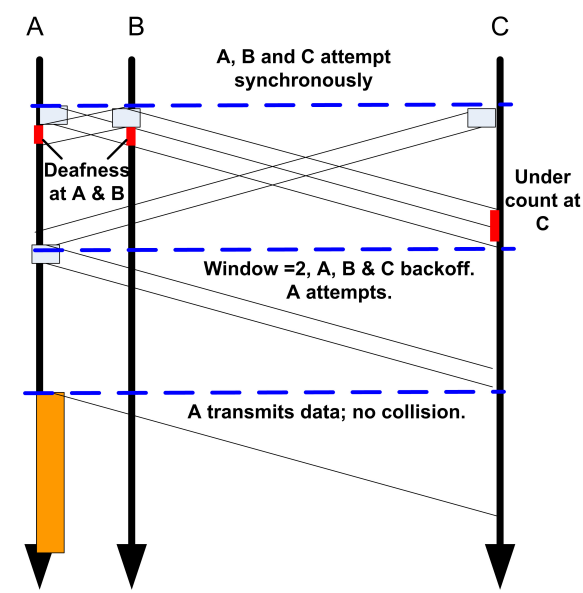

(a)

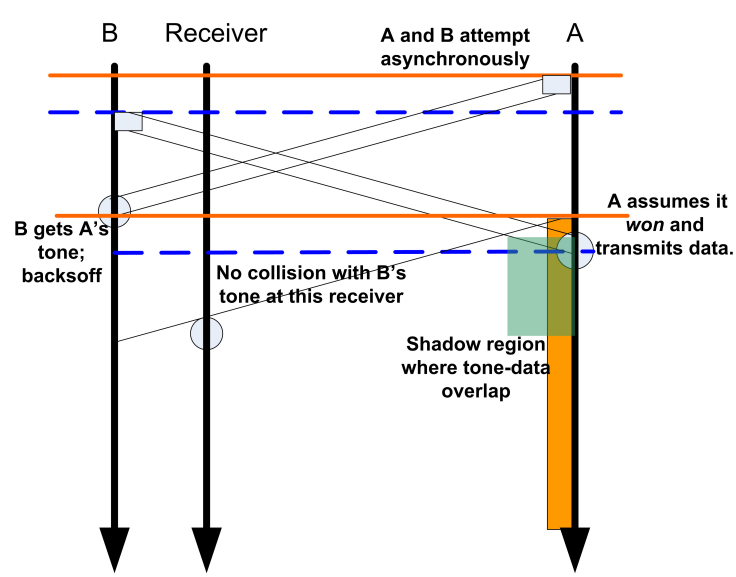

(b)

Fig. 6. Benefit of (a) Higher contention, (b) Aggression and asynchrony.

region, a transmission in aUT-Lohi actually succeeds (because tone and data do not collide) in situations where ST-Lohi and cUT-Lohi would extend the reservation period.

2) Data-Data Collision: Data-data collisions can also occur in T-Lohi if two nodes believe they have won the reservation and so transmit simultaneously.

In ST-Lohi, data-data collisions occur only as a result of bidirectional deafness when reserving nodes are within $D_{\text {deaf }}$ - this condition is necessary and sufficient for data-data collisions. ( $D_{\text {deaf }}$ is quite small for our $T_{\text {detect }}$; in simulations with random node placement only $0.14 \%$ of node pairs are bidirectionally deaf.) Data-data collisions can also occur in aUT-Lohi when pseudo-bidirectional deafness occurs, that is when both tone-data coexistence conditions (Equation 2) and deafness condition (Equation 1) are met. This collision occurs as one node of the pair will assume data reserved because of its aggressive round length, while the other will do the same due to deafness. Such collisions need to be handled at a higher layer using back off and retransmission.

3) Benefit of High Contention: Finally, although we describe collision scenarios above, the presence of an additional contender can solve these situations by effectively extending the reservation period.

Figure 6(a) illustrates this effect for ST-Lohi, where contending nodes A and B are within each other's deaf region. In this case, bidirectional deafness would normally cause both nodes to send data packets that would then collide. However, addition of a third contender $\mathrm{C}$ causes both $\mathrm{A}$ and $\mathrm{B}$ to detect another contender. All nodes backoff and prevent an incorrect data reservation. If this backoff places A and B in separate CRs, then no collision will occur. Similarly additional contenders also "break" the pseudo bidirectional deafness of aUT-Lohi and prevent packet collisions.

\section{Basic Performance Evaluation}

We next evaluate basic performance of T-Lohi through simulation. We begin by looking at the design tradeoffs between its three flavors. We also evaluate important MAC metrics such as throughput, energy efficiency, and fairness.

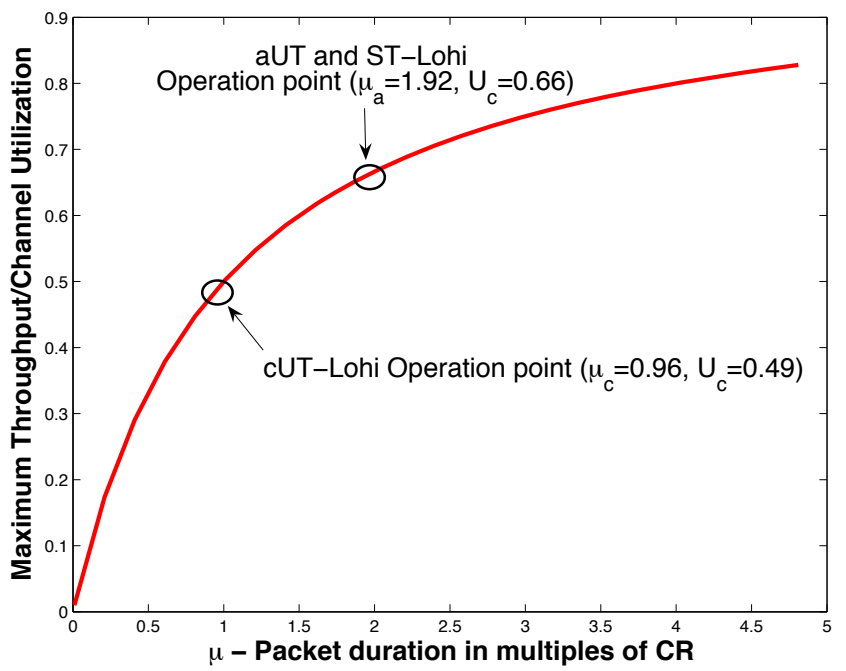

Fig. 7. Maximum theoretical utilization for T-Lohi protocols as $\mu$ is varied, showing the operational points for our simulations.

Simulation results show that T-Lohi achieves better throughput $(34-50 \%)$ than prior published results $(22 \%)$ of other throughput-efficient, underwater MAC protocols [15], [11], while maintaining energy efficiency comparable to UWANMAC [6]. Finally, we quantify the impact of the unique characteristics in acoustic medium access, such as deafness and contender counting, on performance.

\section{A. Simulation Methodology}

We develop a custom acoustic network simulator based on a prior model for underwater time synchronization [16]. (The simulator and simulations are available for download from the authors' website.) We do not currently model packet loss due to channel noise and multi-path, and mainly focus on protocol behavior. Exploration of these effects of is an important direction for future work.

Our default simulation parameters are randomly deployed nodes in a $300 \times 400 \mathrm{~m}$ area. The network is fully connected 


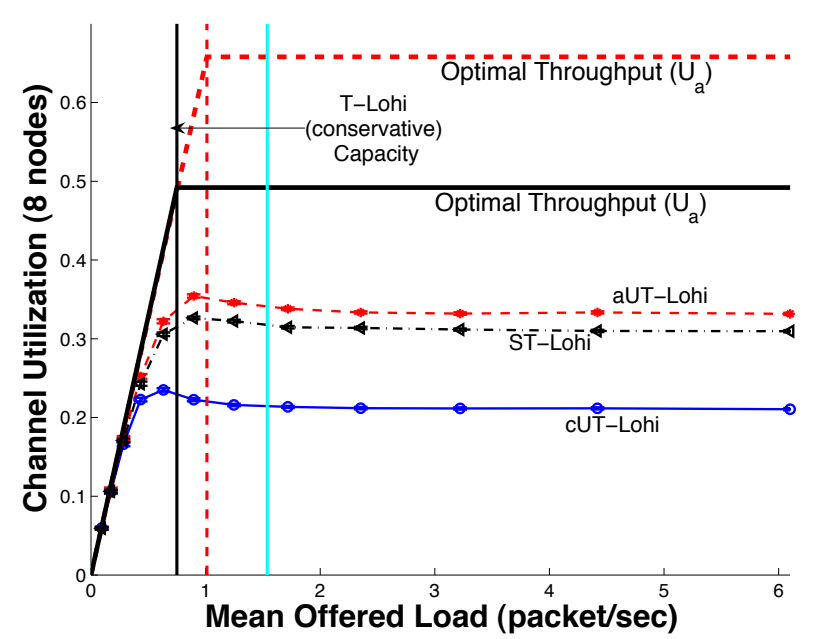

(a) Eight Node Network

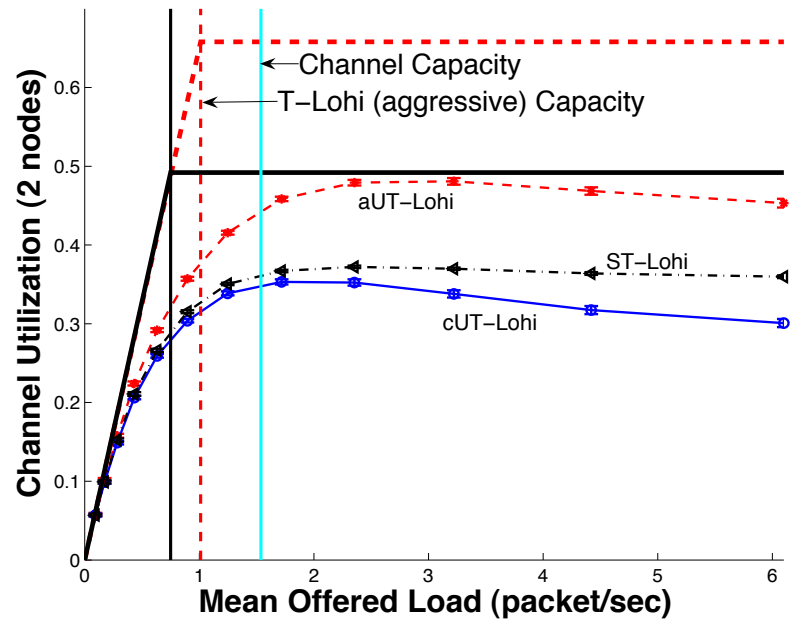

(b) Two node network

Fig. 8. Channel utilization of three T-Lohi flavors. The vertical lines show the channel capacity and the protocol capacities, in packets/s.

with acoustic transmission range of $500 \mathrm{~m}$. Transmit data rate is $8 \mathrm{~kb} / \mathrm{s}$, with 650 -byte packets, implying that packet transmission duration $P_{t x}$ is $650 \mathrm{~ms}$. Tone detection time is $5 \mathrm{~ms}$. Each simulation lasts 100 s and repeats 500 times. We show the mean and $95 \%$ confidence intervals in each graph, but in most cases confidence intervals are barely visible. Nodes transmissions follow a Poisson distribution, one packet at a time, with no queueing. In Section V-C we change to 5 packet transmit bursts with infinite queues. We reevaluated selected earlier results with infinitely deep queues and found T-Lohi performance changed very little.

\section{B. Network Throughput}

Our first goal is to understand how throughput is affected by changes in offered load, network density, and protocol choice. Throughput is an important metric in acoustic communication because of the very limited bandwidth. We first define the maximum theoretical throughput for T-Lohi, assuming perfect scheduling, to provide a performance goal. In this ideal case, there is only one contender per frame, and all T-Lohi RPs will consist of a single contention round (CR).

With perfect scheduling, the best possible throughput is the ratio of data to frame length: $P_{t x} /\left(P_{t x}+C R\right)$. To divorce achievable throughput from a particular topology or hardware, we normalize by $\mu=P_{t x} / C R$, the packet transmission time in multiples of contention rounds. T-Lohi's maximum throughput is then:

$$
\text { TH } \max =\mu /(\mu+1)
$$

Figure 7 shows how the best possible performance varies with $\mu$. In simulation, we send fixed amount of data in each packet (650B), but variation in the duration of the contention round means that aUT- and cUT-Lohi have different achievable performances. This figure also shows the operational points we use in our simulations with a fixed data size; other points on this curve could also be used. For these parameters, the best possible utilization, $U$, is $U_{a}=0.66$ for ST- and aUT-Lohi, and $U_{c}=0.49$ for cUT-Lohi.

1) Throughput as Load Varies: We first examine how the throughput of T-Lohi responds to varying offered load. We expect T-Lohi to be throughput stable because it can detect and count contenders.

Figure 8(a) shows channel utilization as a function of aggregate offered load. The figure also shows two theoretical limits while operating at $\mu_{a}$ and $\mu_{c}$. First, the vertical lines show limits on the offered load due to channel and protocol capacities. Second, we also plot the optimal utilization curves for T-Lohi as the load varies.

We have three observations from this simulation. First, TLohi is very efficient at low offered load, where contention rates are low. When the load is less than 0.5 packets/s, TLohi is very close to the maximum theoretical utilization. Second, as offered load approaches the practical capacity (0.51 packet/s), we see T-Lohi reaches about $50 \%$ of maximum utilization. Finally, as offered load exceeds practical capacity (more than 1 packet/s), we observe that T-Lohi throughput remains stable.

As Figure 9 shows (for ST-Lohi; both aUT and cUT-Lohi exhibit similar curves), the duration of reservation period doubles when the offered load increases between $0.5-1$ packet/s, resulting in the decreased throughput. This figure can also be construed as the MAC delay or latency, which in this case is independent of offered load. Furthermore, Figure 9 also indicates that the reason for stable throughput is the near constant reservation period duration. Thus the combination of contention detection and load-influenced contention counting allows makes throughput stable and load-independent.

2) Impact of Protocol Choice on Throughput: To observe how different protocol design (Section III-B) affects channel utilization, we next compare the three T-Lohi flavors.

Figure 8 shows the channel utilization of T-Lohi flavors at two different network densities. We first observe that cUT-Lohi has saturation capacity about two-third of aUT-Lohi, primarily because of its longer CR length. Although cUT-Lohi has a contention round that is twice that of aUT-Lohi, its capacity is not halved. This disparity is due to the non-linearity of achievable utilization as predicted by Equation 3 .

More interesting is that aUT-Lohi always achieves higher utilization than ST-Lohi (slightly higher with 8 nodes and 


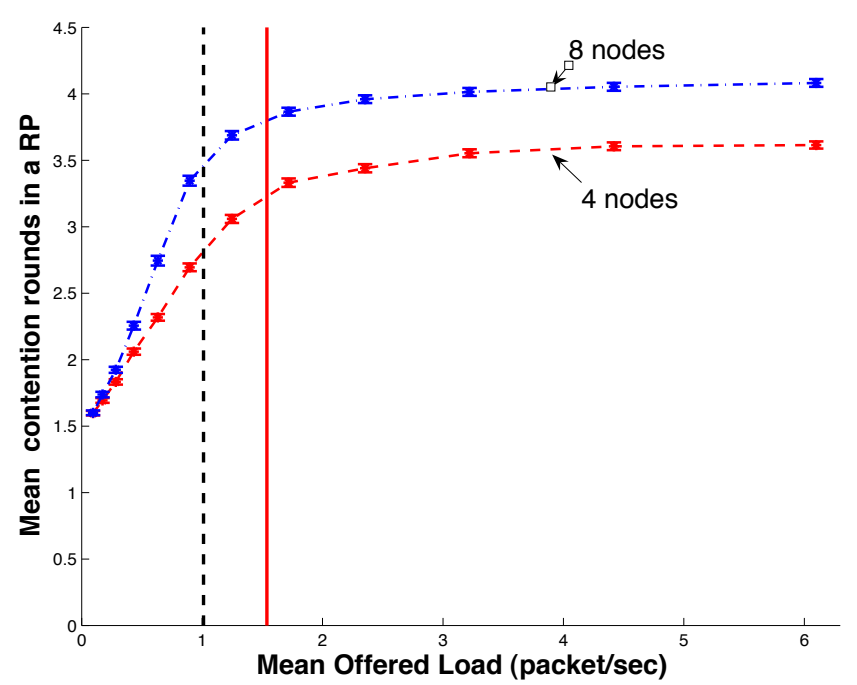

Fig. 9. Average number of contention rounds in a reservation period for ST-Lohi.

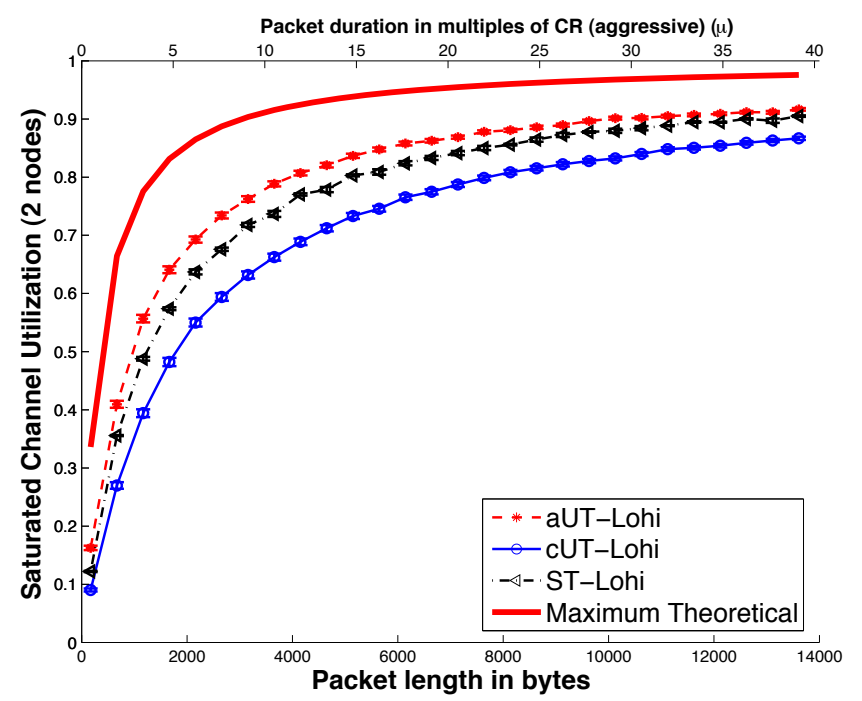

Fig. 10. Channel utilization as $\mu$ is varied by changing the packet length.

much better with only 2 nodes). This result is due (except at low densities, which we explain next) to the slotted access in ST-Lohi that delays all access attempts to the start of the next slot. When both have the same CR $\left(C R_{a U T}=C R_{S T}\right)$, this delay (on average half $\mathrm{CR}$ ) results in greater reservation latency for ST-Lohi. In summary, all the T-Lohi flavors have similar throughput behavior, but ST-Lohi and aUT-Lohi offer higher throughput than cUT-Lohi due to their smaller CRs.

3) Impact of Network Density and Packet Length on Throughput: We next explore how network density and packet length affect T-Lohi's throughput. The throughput of traditional wireless MACs degrades with density, but we expect TLohi to remain stable based on the results from Section IV-B1.

Comparing Figures 8(a) and 8(b), we observe that utilization is significantly lower for aUT-Lohi and cUT-Lohi in denser networks (compared to a 2 node network). In fact the decrease by nearly $15 \%$ is evident even at 4 nodes (not shown here) and does not vary significantly for higher densities. Utilization of

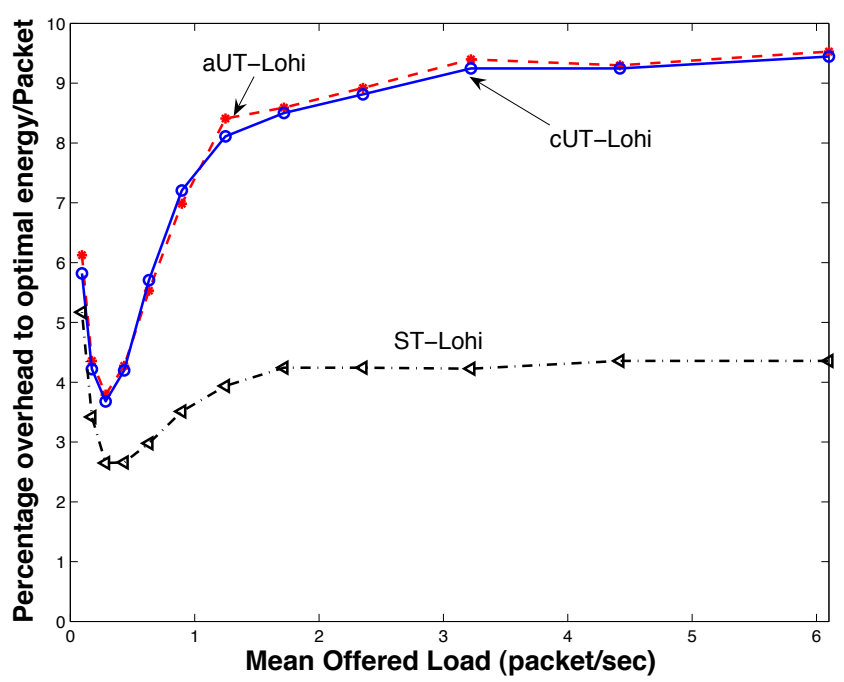

Fig. 11. Relative energy overhead for T-Lohi for an 8 node network

TABLE I

Acoustic MOdem Power Draws

\begin{tabular}{|l|ll|}
\hline Mode & Data & Wake-up Tone \\
\hline Transmit (Max) & $2 \mathrm{~W}$ & $2 \mathrm{~W}$ \\
Receive & $20 \mathrm{~mW}$ & $0.5 \mathrm{~mW}$ \\
Idle/Listen & $20 \mathrm{~mW}$ & $0.5 \mathrm{~mW}$ \\
\hline
\end{tabular}

ST-Lohi, however, does not show such dependency on network density. We have separately evaluated T-Lohi throughput at higher densities (16 and 32 nodes), but we observe no significant differences in throughput curves there.

The higher throughput with two nodes is explained by a combination of asynchrony and the mechanism to handle spatial unfairness. With two nodes and asynchronous access, the quiet period after successful transmission (Section III-B), allows the two nodes to repeatedly swap the channel with just a single CR per frame. However, the similar effect does not often occur in ST-Lohi because of slotted transmission times. In Figure 1(b), only node A contends in slot 3, since $\mathrm{B}$ remains quiet in slot 3 to promote fairness. Nodes that are further away, such as C or D, start contending in slot 4 (not shown in figure) along with $\mathrm{B}$ whose quiet period would have ended. With more than two nodes, this channel swapping is not possible with either flavor of Lohi, since more than one CR will be required.

We also varied $\mu$ using longer packet length, and observed (Figure 10) that the throughput increases monotonically with packet length or $\mu$. Furthermore, under all operating regimes the utilization achieved by T-Lohi remains within 35\% of the theoretical optimal given by Equation 3 .

\section{Energy Efficiency}

Since underwater sensornets are often energy constrained, we next consider the energy efficiency of T-Lohi under varying loads. We expect T-Lohi to be energy efficient because wake-up tone detection reduces the energy cost of long data reservation periods. The modem power values used in our simulations are shown in Table I and roughly match the 


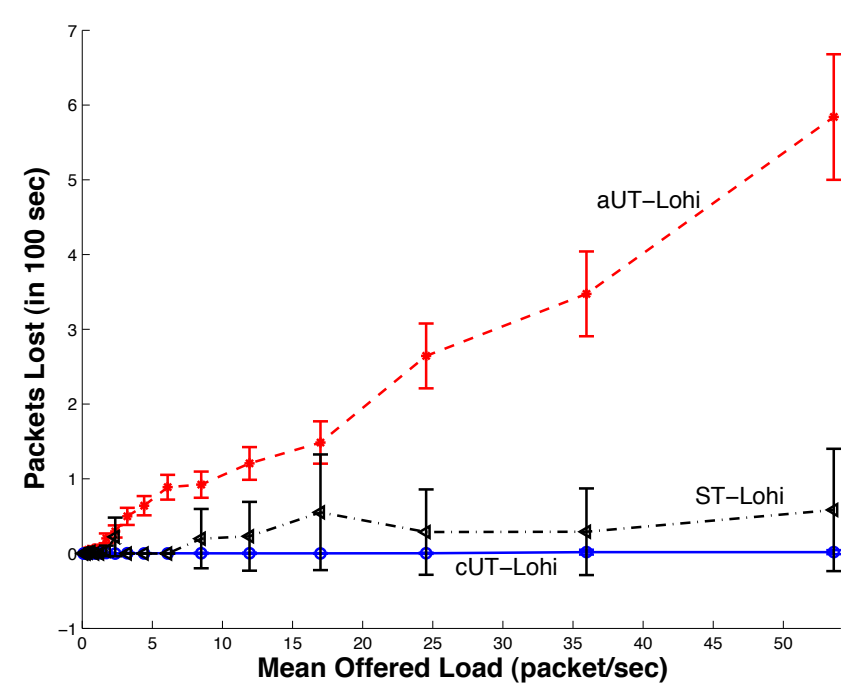

(a) 2 node network

Fig. 12. Packets lost in a fixed duration as offered load is varied

power consumption of our acoustic modem with wake-up tone support [4].

Figure 11 shows the percentage energy overhead of T-Lohi in an eight-node network. We define energy overhead as the cost beyond the optimal energy per packet used in transmitting and receiving a single packet. All protocols are very efficient under all loads, with energy overhead at most $9 \%$ over the optimal cost. ST-Lohi has a very low and nearly constant energy overhead (just $4 \%$ over the optimal) because it prevents any data collision. The overhead is solely due to the cost of sending and receiving tones during the contention rounds. The energy cost of aUT-Lohi increases marginally at higher loads (9\% over optimal at high load versus $4 \%$ at low load) due to data collisions caused by its aggressive policy.

More interestingly, aUT-Lohi and cUT-Lohi have similar energy overhead. While aUT-Lohi gets more packets through than cUT-Lohi, the latter sleeps for longer periods, so the energy cost per packet becomes similar under the Poisson traffic model. For lower network density (4 nodes) cUT-Lohi is about $40 \%$ more energy efficient than aUT-Lohi. The reason can be explained from results in next section where we show that higher density reduces the probability of packet loss for aUT-Lohi.

\section{Protocol Correctness: Impact of Deafness and Aggression}

We now evaluate the impact of deafness and aggressive contention on T-Lohi. Deafness and aggressive contention can cause protocol incorrectness (Section III-C), where multiple nodes believe they have reserved the channel. We quantify the impact of these factors by measuring packet loss over a fixed interval as offered load varies.

Figure 12(a) allows us to make several interesting observations for a two node network. First, cUT-Lohi experiences practically no collision at any offered load. ST-Lohi has very few packet losses but shows high variability, while packet loss for aUT-Lohi increases proportionally to the network load. Investigation of packet loss in aUT-Lohi reveals that,

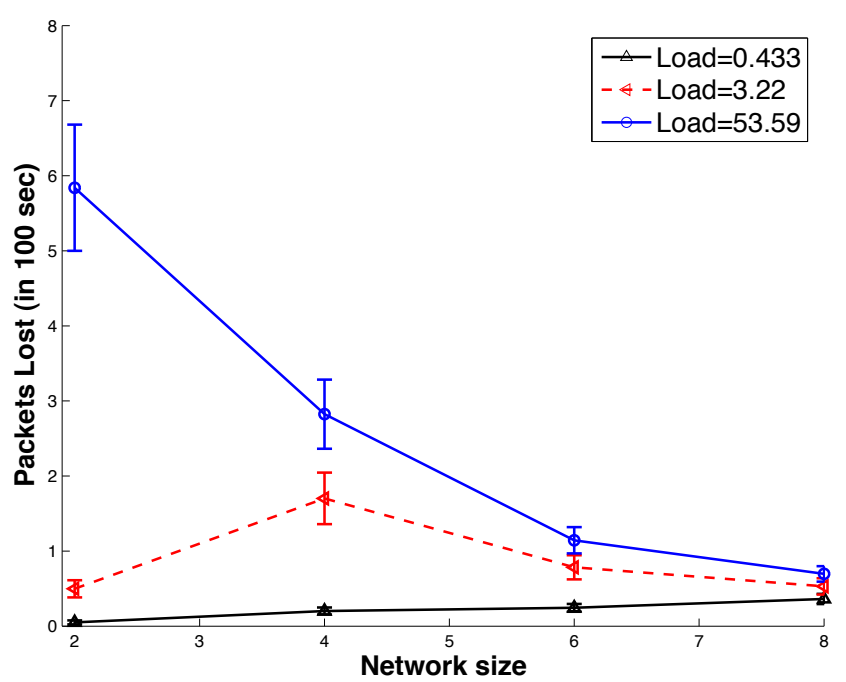

(b) aUT-Lohi with $>2$ nodes

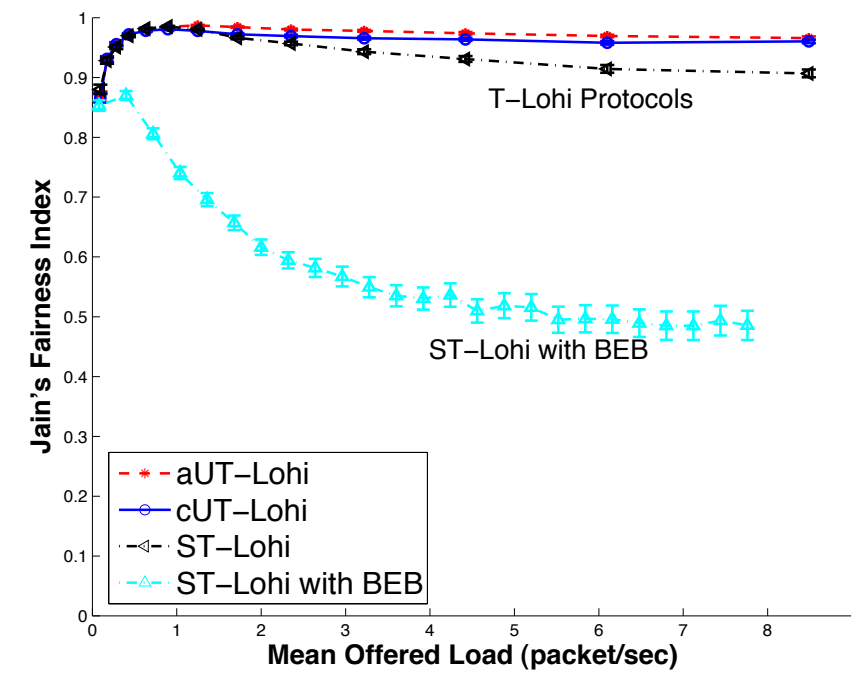

Fig. 13. Jain's fairness index for T-Lohi that can count contender vs. a MAC that can only detect contenders and uses BEB.

in most cases, loss is due to data-data collisions, and that such collisions become more likely at higher load due to its aggressiveness.

The results of packet loss for both cUT-Lohi and ST-Lohi show very little variation over all network densities (omitted here). In Figure 12(b), we see that in aUT-Lohi, the number of losses and its variance decrease as more nodes contend, because more nodes easily break the pseudo-deafness conditions necessary for data-data collision. These results show that under high contention, the impact of both deafness and aggression (in aUT and ST-Lohi) becomes negligible. Meanwhile, cUTLohi provides the most reliable data transfer, especially for sparse and low traffic networks.

\section{E. Impact of Contender Detection and Counting}

T-Lohi exploits space-time uncertainty to provide contention detection (CTD) and counting (CTC). Here we separate these 
capabilities to quantify the impact of contender counting on fairness.

To evaluate the benefits of channel observation we compare T-Lohi with contender counting to a modified version that can only detect (but not count) contention and thus uses binary exponential backoff (BEB). Since systems with collision detection exhibit throughput stability even at high loads (as in Ethernet [17]), here we focus on fairness. Binary backoff provides insufficient information to easily provide fairness. TLohi's contender counting allows for a traffic-adaptive backoff mechanism (Algorithm 1).

We use Jain's fairness index defined as $\frac{\left(\Sigma x_{i}\right)^{2}}{\left(n \cdot \Sigma x_{i}^{2}\right)}$ where $x_{i}$ is the number of packets successfully sent by a node, and $n$ represents the number of nodes in the network. Figure 13 shows the result for an experimental setup consisting of eight nodes run for 500 s to strenuously test protocol fairness. We first observe that the T-Lohi protocols exhibit a high fairness index ( 0.9 and above) that remains nearly constant across all offered loads. In comparison the version employing BEB instead of using contention count for backoff shows an exponential decay in its fairness index. The reason for traffic independent fairness in T-Lohi is again the ability to backoff based on an accurate view of the current congestion level.

\section{F. Future Evaluation}

Although we explore a number of factors above, two remain to be studied: channel effects and multi-hop operation. Our evaluation of these additional factors is ongoing, but we next provide brief, preliminary speculations as to their effects.

Of course, underwater channels have many sources of noise. For data packets, noise typically causes packet loss due to corruption; for tone transmission noise can result in false tones or tone loss. We do not consider packet loss in this paper, however one could add an ARQ mechanism to our protocol (for example, see Stojanovic [18]). In this paper, we expect that loss of data packets will lower effective throughput.

We are currently evaluating the effects of noise on tones (both false positive tones and tone loss). We believe that false detection of tones simply prolongs the reservation period as the tone is consider an indication of contention. For low to moderate rates of false detection, T-Lohi will work correctly albeit with lower throughput. Numerical analysis of reservation period using the T-Lohi contention resolution mechanism shows that with a $50 \%$ chance of a false tone (in each CR), reservations periods require less than two additional rounds to converge (from 3.5 to 4.9 rounds per RP), although with nearly constant false tones convergence becomes unacceptable (for example, 95\% chance of false tones requires $32 \mathrm{CR}$ per RP). Tone loss, on the other hand, could result in incorrect reservations. However, data-data collisions will occur only if the tone is lost in the final round of contention with exactly two contenders (in other cases, contention continues in future rounds due to other tones). For these reasons, we expect that the performance degradation due to moderate channel noise will be small. In addition, the SNUSE modem targets relatively short range communication $(500 \mathrm{~m}$ or less), so some forms of channel noise will be less likely than with longer range modems [1].
One specific class of channel noise is multi-path reflections. Our simulator does not currently consider them; we need to evaluate their effects. We do anticipate that tone echoes ("self"-multipath) could prolong T-Lohi reservation periods. We are currently studying this open question and approaches to mitigate these self-reflections.

Finally, our simulations here consider only a fully connected network. We wish to fully understand single hop characteristics of our MAC before extending it to a multihop network. In future work we expect to explore multi-hop options and effects, including transmit power control, data and control pipelining, and hidden-terminal effects.

\section{Evaluation of Design Alternatives}

After having evaluated the protocol performance using simulation, we now evaluate protocol design alternatives like the duration of contention round and different T-Lohi flavors. We also compare T-lohi's throughput and energy efficiency with a few canonical protocols.

\section{A. Choice of Contention Round Duration}

We first investigate the impact of the duration of contention round $(\mathrm{CR})$ in T-Lohi, as this parameter limits the throughput.

While the round-trip time is selected in cUT-Lohi (Section III-B2) to guarantee collision avoidance, the choice of maximum propagation delay as CR duration for aUT-Lohi seems arbitrary. Moreover, our research on adapting slotted ALOHA to underwater acoustic environment shows transmission slots with additional guard bands (quiet time after data) achieves higher throughput [7]. Another recent MAC protocol for UWSN similarly advocates the benefit of ignoring worst case delays since such scenarios are rare [15]. We believe that although the throughput would increase with shorter contention durations, the stability and energy efficiency is likely to decrease (due to more data collisions).

Figure 14 compares the throughput and energy conservation of the unsynchronized version of T-Lohi with different contention round durations (for reasons of clarity and space we omit similar analysis and results for ST-Lohi). We examine a wide set of contention durations (between 0.1 to 2 times the maximum delay, with a granularity of 0.1 ); here we show specific cases to represent general trends. The throughput performance (Figure 14(a)) of the protocol is maximized at a CR duration equal to half of the maximum propagation delay. Any shorter CR duration lowers throughput and reduces throughput stability at high loads. On the other hand, reducing $\mathrm{CR}$ to below the maximum propagation delay (the aUT-Lohi case) increases energy overhead (Figure 14(b)). We see that the energy overhead nearly quadruples when CR duration is half of the maximum delay, with further increase for shorter CR durations (not shown here).

These results are explained by noting that while a shorter CR duration results in less per packet overhead (and therefore higher throughput), it also increases the probability of datadata collision as a result of incorrect reservations (lower throughput). The energy cost, on the other hand, only increases with a shorter CR duration (due to higher collision 


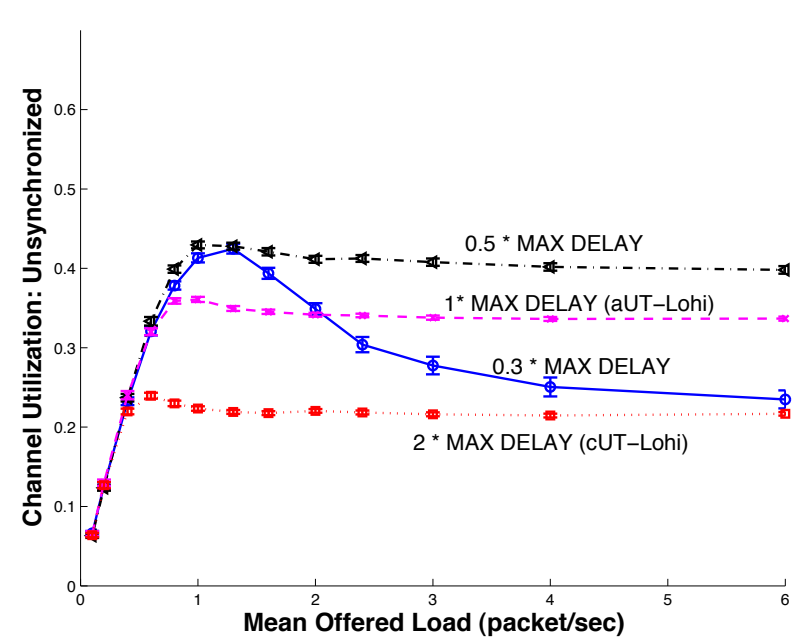

(a) Throughput

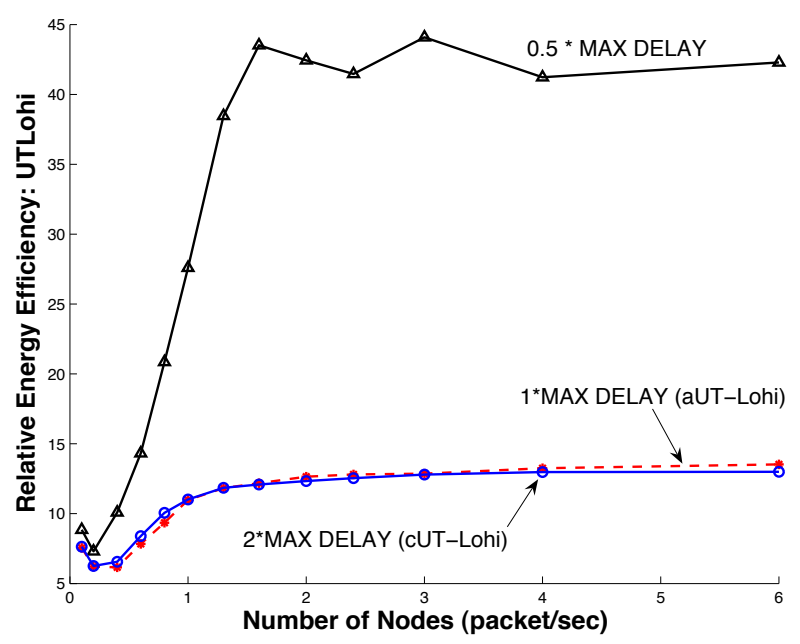

(b) Relative Energy Efficiency

Fig. 14. Impact of different contention round length on Unsynchronized T-Lohi

probability). The reason for similar energy efficiency of aUTand cUT-Lohi was discussed in more detail in Section IV-D.

Conclusion: These results show that our choice for the contention round duration faithfully fulfills the design goals of T-Lohi for both energy and throughput efficiency. Other values might improve throughput, but cannot provide correctness guarantees and thus result in unacceptable amount of packet and energy losses.

\section{B. Comparison of T-Lohi Flavors}

We next compare the T-Lohi flavors (ST-Lohi, cUT-Lohi, and aUT-Lohi). Table II encapsulates our evaluation of these protocols for a few important MAC attributes that are part of our initial design.

ST-Lohi performs well, but slot synchronization increases implementation complexity. While cUT-Lohi has lower implementation complexity, it also has lower throughput. On the other hand, aUT-Lohi shows good performance for all measurements, with a slight degradation on correctness (prevention of any collision) due to its aggressive contention policy. However, such correctness issue becomes less of a concern at higher densities, since more contending nodes break the collision conditions (Section IV-D).

Therefore, we conclude that the aggressive unsynchronized T-Lohi (aUT-Lohi) is the best choice for general applications. We next use aUT-Lohi to represent the T-Lohi class of MAC protocols when comparing with other protocols.

\section{Comparison with existing MAC protocols}

We next compare T-Lohi to three canonical medium access mechanisms one might consider for underwater use: TDMA, CSMA and ALOHA. We focus on how throughput and energy efficiency compare in different scenarios.

1) Comparative Protocols Parameters: TDMA works well in some networks, although its synchronization often requires centralized or complex coordination. We allocate TDMA slots to senders, with slot duration equal to packet transmission time plus the maximum propagation delay (similar to an implementation by a group at MIT [19]). The additional wait time is required to guarantee collision free reception of a TDMA transmission.

ALOHA provides an opposite extreme, with a very simple, fully distributed MAC. For the underwater acoustic environment, we compare with a modified version of slotted ALOHA that increase slot duration beyond packet length (with guard bands) to reduce collisions, an extension that is important for high-latency networks [7]. We select guard band duration to maximize throughput in our simulations from empirical evaluations.

In RF, carrier sensing or CSMA significantly improves throughput compared to ALOHA. With high-latency acoustic networks, space-time uncertainty makes sensed channel state less reliable. Our implementation of CSMA is relatively simple, and is similar to the one used in Seaweb [20]. Nodes transmit if channel is sensed clear at that instant; if not they backoff uniformly within the maximum propagation delay to attempt later.

We now compare the throughput and energy overhead of aUT-Lohi with TDMA, CSMA, and ALOHA. We expect that TDMA will provide the best throughput near saturation, while CSMA and ALOHA performance will degrade at higher loads. Traditional protocols do not attempt to reduce energy consumption, so we expect T-Lohi to be much more energy frugal. We keep the same simulation parameters as Section V-A, but for two. Here we consider a very dense, 32-node network. We also adopt a traffic model with a 5 packet burst, where the mean offered load is the same as in previous simulations.

2) Comparing Channel Utilization: Figure 15 shows the channel utilization of the protocols under bursty traffic. As expected utilization at high load is best with TDMA, since when all nodes are saturated, round-robin is the best possible policy. However, at low to medium loads (until 1 packet/s), before the channel starts to saturate, aUT-Lohi provides higher channel utilization because contention-based channel access provides lower latency. In addition, both aUT-Lohi and TDMA are stable when the channel is overcommitted. By comparison, utilization for CSMA and our modified slotted ALOHA drops 
TABLE II

TABLE COMPARING THE PERFORMANCE OF T-LOHI FLAVORS

\begin{tabular}{|l|lllll|}
\hline T-Lohi Flavor & Throughput & Energy Efficiency & Fairness & Correctness & Complexity \\
\hline ST-Lohi & Good & High & High & High & High \\
cUT-Lohi & Low & High & High & High & Low \\
aUT-Lohi & Good & High & High & Good & Low \\
\hline
\end{tabular}

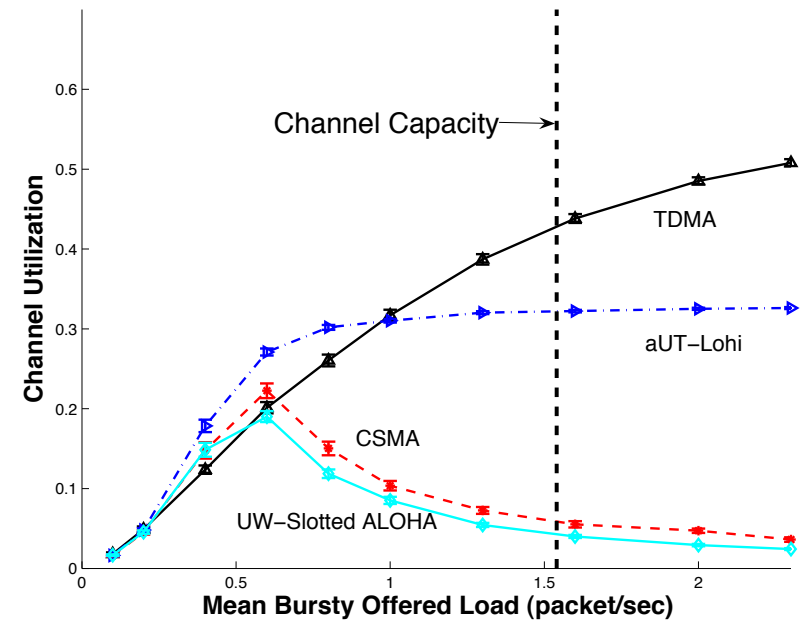

(a) Throughput

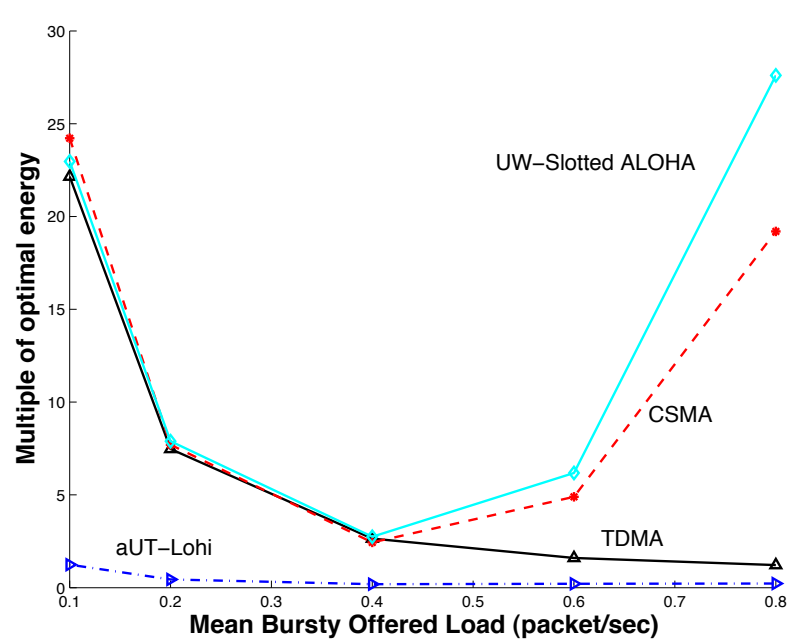

(b) Relative Energy Efficiency

Fig. 15. Comparison of different MAC approaches with aUT-Lohi with bursty traffic and infinite packet queuing (the graphs consider slightly different ranges of offered load).

when offered load exceeds one-third of channel capacity, and asymptotically approaches zero.

3) Comparing Energy Efficiency: Figure 15(b) shows the energy consumed in sending a single packet as a multiple of the optimal energy. We make several observations for different protocols. First, aUT-Lohi shows a constant, load-independent energy-overhead that is lower than other protocols. Second, at low loads, other protocols show a large energy overhead due to idle listening. Finally, while energy overhead in TDMA decreases as load increases, the energy consumed by CSMA and modified slotted ALOHA falls first and then rises significantly. TDMA does well because it provides stable, high throughput at high loads by eliminating collision (Figure 15). In contrast, collisions at even moderate loads cause poor performance and unstable energy usage in CSMA and modified slotted ALOHA.

TDMA, CSMA and ALOHA are not designed for energy efficiency, and we have not tried to optimize their energy consumption. Improvements to their basic approaches, such as using wake-up tones before data, could greatly improve their energy efficiency. However, while this approach would help TDMA by a constant factor, it cannot address the inefficiency of CSMA and ALOHA at high loads that lead to more data collisions.

\section{RELATED WORK}

Sensor networks have raised awareness of the the importance of energy efficiency. We have previously argued [8], that current RF approaches [9], including low-power listening, scheduling, or combinations (as in B-MAC, S-MAC, SCPMAC, or variants) are insufficient to cope with the propagation delay of an acoustic network. In contrast, T-Lohi exploits our low-power tone receiver in the MAC to conserve energy in spite of latency. There have been other terrestrial MAC protocols, such as BTMA [21] and DBTMA [22], that use busy tones to deal with the hidden terminal and exposed terminal problems. These protocols, however, assume separate channels for tones and data, and do not consider large propagation delays as they are designed for RF networks.

Satellite networks, like acoustic networks, cope with large propagation delays. However, their design is usually simplified by coordination with a single visible satellite [23]. T-Lohi, instead, is fully distributed without a central coordinator.

Most closely related are other MAC protocols designed for underwater acoustic networks. Early work proposed CSMA with RTS/CTS, resulting in low throughput [20]. Other work employs CDMA by developing code distribution techniques [24], but at high energy cost. Rodoplu and Park extend S-MAC's schedule synchronization to sender-receiver pairs underwater [6]. It improves energy efficiency, but lacks latency-tolerant contention resolution, and so is only suited for applications that have extremely low traffic rates. S-FAMA uses an RTS/CTS exchange to prevent collisions, with an RTT penalty per packet attempt [11]. Peleato and Stojanovic extend this work using the fact that inter-node distance is seldom the maximum transmission range, allowing less than RTT penalty per packet [15]. This approach is promising and, unlike our work, addresses multi-hop deployments. However, this approach does not minimize energy consumption, provides relatively low throughput (less than 20\%), good stability and flexibly adapts to many applications. 


\section{CONCLUSIONS}

In this paper we leveraged the opportunities in acoustic medium access along with low power wake-up tone hardware to design T-Lohi, a new class of energy efficient, stable and flexible MAC protocols for UWSN. We describe three flavors of T-Lohi representing different design choices, and compare them to traditional CSMA, TDMA, and ALOHA. We show that T-Lohi provides both good throughput and energy efficiency.

\section{REFERENCES}

[1] J. Heidemann, Y. Li, A. Syed, J. Wills, and W. Ye., "Underwater sensor networking: Research challenges and potential applications," in Proceedings of the IEEE WCNC, Las Vegas, Nevad, USA, Apr. 2006.

[2] J. Partan, J. Kurose, and B. N. Levine, "A survey of practical issues in underwater networks," in Proceedings of the WUWNet '06, 1st ACM workshop on Underwater networks. New York, NY, USA: ACM Press, 2006, pp. 17-24.

[3] Micro-Modem Overview, Woods Hole Oceanographic Institution, http: //acomms.whoi.edu/micromodem/.

[4] J. Wills, W. Ye, and J. Heidemann, "Low-power acoustic modem for dense underwater sensor networks," in Proceedings of WUWNet05. Los Angeles, California, USA: ACM, September 2006, pp. 79-85.

[5] "Tagging of pacific pelagic project," http://www.toppcensus.org/.

[6] V. Rodoplu and M. K. Park, "An energy-efficient MAC protocol for underwater wireless acoustic networks," in Proceedings of the IEEE OCEANS'05 Conference, Sep. 2005.

[7] A. Syed, W. Ye, B. Krishnamachari, and J. Heidemann, "Understanding spatio-temporal uncertainty in medium access with aloha protocols," in Proceedings of WUWNet06. Montreal, Quebec, Canada: ACM, September 2007.

[8] A. A. Syed, W. Ye, and J. Heidemann, "T-Lohi: A new class of MAC protocols for underwater acoustic sensor networks," in To appear in Proceedings of the IEEE Infocom, Pheonix, AZ, Apr. 2008.

[9] K. Kredo II and P. Mohapatra, "Medium access control in wireless sensor networks," Comput. Networks, vol. 51, no. 4, pp. 961-994, 2007.

[10] Benthos, Inc., Fast And Reliable Access To Undersea Data, http://www. benthos.com/pdf/Modems/ModemBrochure.pdf.

[11] M. Molins and M. Stojanovic, "Slotted FAMA: A MAC protocol for underwater acoustic networks," in Proceedings of the IEEE OCEANS'06 Asia Conference, Singapore, May 2006.

[12] V. Bharghavan, A. Demers, S. Shenker, and L. Zhang, "MACAW: A media access protocol for wireless LAN's," in Proceedings of the ACM SIGCOMM Conference. London, UK: ACM, Sep. 1994, pp. 212-225.

[13] L. Chen, S. H. Low, and J. C. Doyle, "Random access game and medium access control design," Dec. 2006, unpublished manuscript available at http://www.cds.caltech.edu/ chen/papers/ramac.pdf.

[14] K. Sanzgiri, I. D. Chakeres, and E. M. Belding-Royer, "Determining intra-flow contention along multihop paths in wireless networks," in Proceedings of BROADNETS'04. IEEE Computer Society, 2004, pp. 611-620.

[15] B. Peleato and M. Stojanovic, "A MAC protocol for ad-hoc underwater acoustic sensor networks," in Proceedings of the WUWNet '06. New York, NY, USA: ACM Press, 2006, pp. 113-115.

[16] A. Syed and J. Heidemann, "Time synchronization for high latency acoustic networks," in Proceedings of the IEEE Infocom, Barcelona, Catalunya, Spain, Apr. 2006.

[17] J. Wang and S. Keshav, "Efficient and accurate ethernet simulation," in LCN '99: Proceedings of the 24th Annual IEEE Conference on Local Computer Networks. Washington, DC, USA: IEEE Computer Society, 1999, p. 182.

[18] M. Stojanovic, "Optimization of a data link protocol for an underwater acoustic channel," in Proceedings of the IEEE OCEANS'05 - Europe Conference, June 2005, pp. 68-73.
[19] I. Vasilescu, K. Kotay, D. Rus, M. Dunbabin, and P. Corke, "Data collection, storage, and retrieval with an underwater sensor network," in Proceedings of ACM SenSys'05, 2005, pp. 154-165.

[20] J. Rice, "SeaWeb acoustic communication and navigation network," in Proceedings of the International Conference on Underwater Acoustic Measurements: Technologies and Results, Heraklion, Greece, Jun. 2005.

[21] F. A. Tobagi and L. Kleinrock, "Packet switching in radio channels: Part II-the hidden terminal problem in carrier sense multiple-access and the busy-tone solution," IEEE Trans. on Computing, vol. 23, no. 12, pp. 1417-1433, 1975.

[22] Z. J. Haas and J. Deng, "Dual busy tone multiple access (DBTMA)-a multiple access control scheme for ad hoc networks," IEEE Transactions on Communications, vol. 50, no. 6, pp. 975-985, 2002.

[23] H. Peyravi, "Medium access control protocols performance in satellite communications," IEEE Communication Magazine, vol. 37, no. 3, pp. 62-71, Mar. 1999.

[24] G. G. Xie and J. Gibson, "A networking protocol for underwater acoustic networks," Department of Computer Science, Naval Postgraduate School, Tech. Rep. TR-CS-00-02, Dec. 2000.

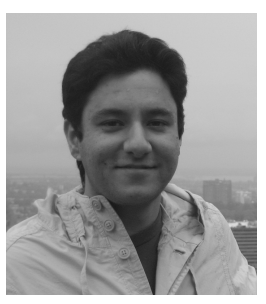

Affan Syed is a fourth year Computer Science $\mathrm{Ph} . \mathrm{D}$. candidate at the Information Sciences Institute (ISI) of USC. At ISI he is part of the ILENSE and SNUSE (Sensor Networks for Undersea Seismic Experimentation) group. His research focus is in underwater acoustic sensor networks. His research interests include MAC layer design, time synchronization, and terrestrial-to-underwater protocol migration. He received his B.S. in Electrical Engineering from National University of Science and Technology, Pakistan and M.S. in Electrical Engineering from USC in 2000, and 2004 respectively. He is a student member of ACM and IEEE, and part of the Phi Kappa Phi Honor society.

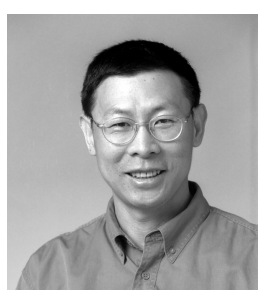

Wei Ye is a Research Assistant Professor at the Information Sciences Institute and the Computer Science Department, University of Southern California. He received his B.S. and Ph.D. degrees in Electrical Engineering from Xidian University, China and his M.S. degree in Computer Science from USC in 1991, 1996 and 2001 respectively. He is working in the area of wireless sensor networks and underwater acoustic networks. His research interest includes MAC layer design, energy management, network architecture, signal processing, experimental testbeds and operational systems for scientific applications. He is an associate editor of the IEEE Transactions on Mobile Computing and an area editor of the ACM Mobile Computing and Communications Review. He is a member of IEEE and ACM.

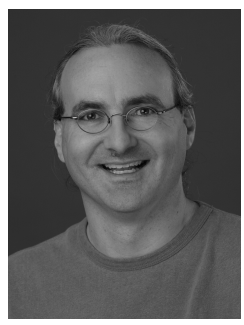

John Heidemann is a Senior Project Leader at USC/ISI and a Research Assistant Professor of Computer Science at USC. At ISI he leads I-LENSE, the ISI Laboratory for Embedded Networked Sensor Experimentation, and investigates network protocols and traffic analysis as part of the ANT (Analysis of Network Traffic) group. He received his B.S. from University of Nebraska-Lincoln and his M.S. and $\mathrm{Ph} . \mathrm{D}$. from UCLA, and is a member of ACM and Usenix. 